Check for updates

Cite this: Nanoscale Horiz., 2020, 5,12

Received 24th May 2019,

Accepted 16th August 2019

DOI: 10.1039/c9nh00347a

rsc.li/nanoscale-horizons

\section{Small-angle X-ray scattering of nanoporous materials}

\begin{abstract}
Samuel S. Welborn (D) ab and Eric Detsi (D) *ab
Small-angle X-ray Scattering (SAXS) is a non-invasive reciprocal space characterization technique that provides statistically representative microstructural information about a material. This focus article examines SAXS as a tool to probe the microstructure of nanoporous materials. We seek to educate the reader on scattering instrumentation and requisite scattering theory pertinent to nanoporous materials, and describe analytical models used to fit SAXS data, to elucidate the microstructure of these nanoporous materials including their morphological features such as the pore size distribution, pore curvature, and specific surface area. Finally, we fit and compare the aforementioned models to real scattering data of nanoporous gold synthesized in our laboratory. The models yield realistic material parameters for the nanoporous gold morphology, in agreement with electron micrograph images. In addition, we have investigated the scattering patterns and characteristic electron micrographs of nanoporous copper and nanoporous antimony also synthesized in our laboratory. The models discussed in this paper do not yield realistic material parameters for specific nanoporous copper and nanoporous antimony morphologies, however. This could be the subject of follow-up work.
\end{abstract}

\section{Introduction}

While the properties of dense macroscopic materials are governed by bulk atoms, many material properties become governed by surface atoms and related phenomena when structural size is on the order of nanometers. In this regime, surface atoms constitute a much larger fraction of the material than bulk atoms. One such high surface-to-volume ratio class of materials corresponds to nanoporous (NP) materials, including NP metals, ${ }^{1-24} \mathrm{NP}$ metal oxides, ${ }^{25-28}$ metal-organic frameworks (MOFs), ${ }^{26,29-35}$ zeolites, ${ }^{29,30,36-39}$ covalent organic frameworks (COFs), ${ }^{40-42}$ carbonaceous materials such as carbide-derived carbon (CDC), ${ }^{43-47}$ and porous polymer networks (PPNs), ${ }^{48,49}$ all characterized by open channels or pores classified according to the IUPAC's ranking in micropores (less than $2 \mathrm{~nm}$ ), mesopores (between 2-50 $\mathrm{nm}$ ), and macropores (larger than $50 \mathrm{~nm}$ ). These NP materials have been widely investigated for various applications including gas storage (e.g. $\mathrm{H}_{2}$ storage and $\mathrm{CO}_{2}$ capture), ${ }^{30,35,44,45,50}$ biotechnology, ${ }^{51}$ radiation resistance, ${ }^{52}$ plasmonics, ${ }^{16,53-57}$ (photo)catalysis, ${ }^{17,18,35,38,55,58-66}$ sensing, ${ }^{67,68}$ actuation, ${ }^{8,68-71}$ and energy

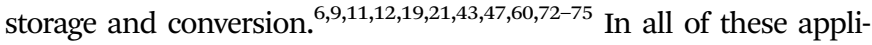
cations, the characteristic size, specific interfacial area, and curvature are critical and necessitate proper quantification.

\footnotetext{
${ }^{a}$ Department of Materials Science \& Engineering, University of Pennsylvania, Philadelphia, PA 19104-6272, USA. E-mail: detsi@seas.upenn.edu

${ }^{b}$ Vagelos Institute for Energy Science and Technology (VIEST), Philadelphia, PA 19104, USA
}

(i) Starting with the characteristic size, usually the average pore size is manually measured from scanning and transmission electron micrographs (SEM and TEM). ${ }^{5,76}$ However, SEM and TEM data only capture a small fraction of the billions of pores within the often voluminous NP materials. Structure sizes deduced from SEM and TEM micrographs, therefore, are not statistically representative of the whole specimen. In an effort to improve upon this limitation, McCue et al. recently developed an automated computational method to calculate average ligament and pore sizes, specific to NP metals, reducing the need for by-hand measurement. ${ }^{77}$ While useful, this method still relies on incomplete data from electron micrographs. ${ }^{5,76}$ (ii) Next, in terms of specific surface area, even though porosimetry techniques such as nitrogen adsorption (BET) are commonly used to determine the specific surface area of NP materials, ${ }^{11,13,14}$ these techniques usually require a large amount of porous materials to yield reliable values. In several cases, it is not straightforward to produce a large amount of NP materials for porosimetry studies. In an effort to overcome this limitation, Detsi et al. derived an analytical expression for a direct computation of the specific surface area of NP materials. ${ }^{78}$ Though useful, $^{79-84}$ this analytical model is only valid in specific situations where the size of ligaments and pores in NP metals are comparable. ${ }^{78}$ An effective approach to characteristic size and specific surface areas of NP materials is needed, and SAXS can provide this information. (iii) Finally, in terms of curvature, the surface curvature of a structure (which is associated with the surface free energy) provides a driving force for morphological evolution via the Gibbs-Thomson effect. ${ }^{85,86}$ In order to study 
the curvature of a nanoscale structure, we currently rely heavily on computer models, ${ }^{85,87}$ and other intensive techniques, including X-ray tomography (which cannot resolve nanoscale features). In this article, we expand on a SAXS method capable of providing this information.

SAXS is a reciprocal space technique utilized by researchers in various fields, spanning polymer science, ${ }^{88}$ biology, ${ }^{89}$ metallurgy, ${ }^{90-92}$ and astronomy. ${ }^{93}$ The technique was pioneered by André Guinier, who measured crystallite size in age-hardened $\mathrm{Al}-\mathrm{Cu}$ alloys using the scattering instrument he built for his dissertation, the 'Guinier Camera' ${ }^{90,94,95}$ Guinier, along with colleague Gérard Fournet, wrote a 1955 book on the topic, SmallAngle Scattering of X-rays. The book serves as an excellent general reference for scattering data interpretation, and the reader is encouraged to consult it for theory beyond the scope of this paper. ${ }^{90}$ Guinier's book develops scattering intensity functions for a wide variety of particle shapes (sphere, cylinder, etc.), but the NP materials we are interested in do not fit neatly into these categories; therefore, general models are appropriate. The aims of this focus paper are to: (i) provide those who are interested in this field with useful background information on SAXS techniques and its benefits and limitations; (ii) elucidate the theoretical foundations of SAXS, expand in depth for models related to NP metals, in particular NP gold (NP-Au), and offer insight on the morphology and topology of NP materials using simulated structures; (iii) provide a description of other models used to fit scattering data for a broad range of NP materials and demonstrate its applicability for the determination of characteristic size, and specific surface area.

\section{Scattering instrumentation}

While X-ray diffraction (XRD) measures coherent scattering of atomic planes at wide scattering angles, SAXS can measure much larger feature sizes, on the order of 1-100 nm, in transmission or reflection mode. This reflection mode is commonly referred to as grazing incidence small-angle X-ray scattering (GISAXS), however the present article will focus on measurement in transmission mode (SAXS). If the instrument has a long enough sample-todetector distance, it is capable of probing micron-sized dimensions in what is named Ultra Small-Angle X-ray Scattering (USAXS). Fig. 1 displays a simplified diagram of a typical SAXS instrument in transmission geometry. In the lab-scale SAXS setup shown in Fig. 1a, polychromatic and unfocused X-rays, whose characteristic wavelength is determined by the elemental nature of the source, are generated at point (b). Various monochromation and beam collimation instruments are used after the beam exits the source, encompassed in point (c) in our diagram. After exiting point (c), the X-rays bombard the sample at point (d), and one of three things can happen: they can be scattered, transmitted, or absorbed by the material. When scattered, the interaction between the material's electrons and the incoming X-ray produces a secondary, so-called scattered, wave. ${ }^{96}$ Such an X-ray-electron interaction is typically assumed to be elastic, where the incoming wave's energy and momentum are conserved after the scattering event. ${ }^{90}$

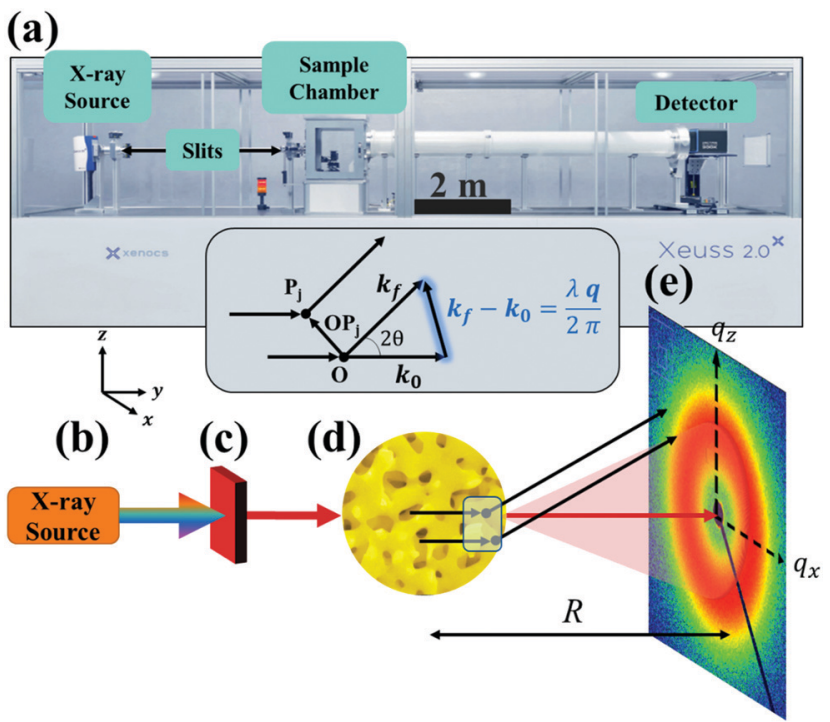

Fig. 1 SAXS instrumentation (a) and geometry ( $b-e)$. X-rays are generated at point (b) and are monochromated and collimated at point (c). The beam bombards the material at point (d), where the X-rays do one of three things: scatter with the electrons in the material, transmit through the material, or absorb into the material. The transmitted beam and secondary scattered waves travel down an evacuated tube of length $\boldsymbol{R}$, and are detected at point (e). In addition, the geometry of the scattering theory described in the theory section is displayed above (d). Both the SEM of the sample (d) and the detector pattern (e) are from NP-Au.

Both the scattered and transmitted X-rays travel the sampleto-detector distance $R$ as illustrated in Fig. 1, and finally interact with the detector at point (e). We display the commonly used $2 \mathrm{D}$ area detector, however this can be replaced by a $1 \mathrm{D}$ line or $0 \mathrm{D}$ point detector. By increasing $R$, the range of accessible scattering angles becomes smaller, resulting in larger realspace dimensions due to the inverse proportionality between real and reciprocal space. Thus, transmission SAXS is limited by the instrumental geometry: to probe length scales pertinent to NP materials, which can have characteristic dimensions of hundreds of nanometers, a large instrument is required. For example, to probe real-space dimensions of $\sim 350 \mathrm{~nm}$ with a Cu source $(\lambda=1.5406 \AA$ ), $s$ needs to be roughly $5 \mathrm{~m}$. Due to this length requirement, performing SAXS with an elemental X-ray source in a lab setting is typically carried out under vacuum to minimize incoherent background scattering by air. Synchrotron radiation mitigates the incoherent scattering issue by making use of high energy and high flux X-rays, and thus SAXS can be performed in air. The reader is referred to sources provided in references ${ }^{90,97}$ for further detailed instrumental information.

\section{Scattering theory}

\subsection{Scattering by a discrete charge distribution}

When X-rays impinge on an atom within a particle, the atom's electrons are forced to vibrate with the radiation's electric field and become emission sources for electromagnetic scattered waves. ${ }^{98}$ In principle, the X-ray's magnetic field also interacts 
with the spin and orbital magnetic moments of the electrons, however the quantity of such scattered waves is negligible in comparison to those originating from the electric field. ${ }^{98}$ For a single atom, this outgoing radiation is emitted in all directions (in the case of an unpolarized X-ray source, spherically). ${ }^{98}$ Its amplitude depends on the atom's electronic structure (i.e. the number of electrons it contains and their spatial arrangement) and the source X-ray's amplitude. The electric field of a scattered wave at a distance $R$ from emitted atomic position $\boldsymbol{P}_{\boldsymbol{j}}$ (Fig. 1) can be described by eqn (1): ${ }^{90}$

$$
E_{j}=\frac{E_{0}}{R} r_{\mathrm{e}} f_{j} \mathrm{e}^{-i \frac{2 \pi}{\lambda}\left(\boldsymbol{k}_{\boldsymbol{f}}-\boldsymbol{k}_{0}\right) \cdot \boldsymbol{O P}}
$$

Here, $E_{0}$ is the electric field amplitude of the incoming wave, $r_{\mathrm{e}}$ is the classical electron radius, $f_{j}$ is the atomic form factor (derived in the Appendix), $\boldsymbol{k}_{\boldsymbol{f}}$ is the unit wave vector after scattering, $\boldsymbol{k}_{0}$ is the unit wave vector before scattering, $\lambda$ is the X-ray's wavelength, and $\boldsymbol{O P}_{\boldsymbol{j}}$ is a vector from an arbitrary origin $\boldsymbol{O}$ and point $\boldsymbol{P}_{\boldsymbol{j}}$. Conservation of energy requires the amplitude to be inversely proportional to the distance from the source, $R$, as the electric field spreads equally around spherical shells of area $4 \pi R^{2}$ with increasing distance from the emission source. The inclusion of the classical electron radius, $r_{\mathrm{e}}=e^{2} / 4 \pi \varepsilon_{0} m_{\mathrm{e}} c^{2}$, where $e$ is the elementary charge, $\varepsilon_{0}$ is the permittivity of free space, and $m_{\mathrm{e}}$ and $c$ are the mass of the electron and speed of light, respectively, ensures proper electric field units. Its inclusion here can be derived from Maxwell's equations. ${ }^{96,98}$ The scattering vector $\boldsymbol{q}$ is defined by the difference between the incoming and outgoing wavevectors as $\boldsymbol{q}=\frac{2 \pi}{\lambda}\left(\boldsymbol{k}_{\boldsymbol{f}}-\boldsymbol{k}_{0}\right)$ and has magnitude $|\boldsymbol{q}|=\frac{4 \pi}{\lambda} \sin (\theta)$. Further in this article, the magnitude $|\boldsymbol{q}|$ will simply be referred to as $q$.

Noting the principle of superposition of waves, we can determine the electric field of all irradiated atoms at a distance $R$ (sample-to-detector distance in Fig. 1) from the source by summing over all atoms within the irradiated volume (eqn (2)). The intensity of the field at the detector positioned at distance $R$ is deduced from its amplitude multiplied by the complex conjugate, $|E|^{2}$ (eqn (3)): ${ }^{90}$

$$
\begin{gathered}
E=\sum_{j} E_{j}=\frac{E_{0}}{R} r_{\mathrm{e}} \Sigma_{j} f_{j} \mathrm{e}^{-i \boldsymbol{q} \cdot \boldsymbol{O P}} \boldsymbol{j} \\
I(\boldsymbol{q})=I_{0} \Sigma_{k, j} f_{k}^{*} f_{j} \mathrm{e}^{i \boldsymbol{q} \cdot\left(\boldsymbol{O P}_{\boldsymbol{k}}-\boldsymbol{O P}_{j}\right)}
\end{gathered}
$$

In eqn (3), $I_{0}=E_{0}{ }^{*} E_{0} r_{\mathrm{e}}{ }^{2} R^{-2}$ absorbs the incident intensity $\left|E_{0}\right|^{2}$, the classical electron radius, and the sample-to-detector distance. Note that this definition holds true for the rest of this article. When the waves in eqn (2) are in phase, (i.e. when there is a repetitive structural size in the irradiated material like atomic planes or, in NP materials, the characteristic pore-pore spacing) they give rise to peaks in intensity at specific values of $\boldsymbol{q}$ (eqn (3)). In this way, the intensity at the detector as a function of the scattering vector $\boldsymbol{q}$ is a Fourier series that relies on the positions of atoms in the sample and their atomic form factors. While these general expressions for a discrete charge distribution are useful in understanding X-ray-electron interactions, we can consider the continuous case for practical matters. In the next section, we will derive a general scattering equation for a continuous charge distribution and apply it later to NP materials.

\subsection{Scattering by continuous charge distribution and notion of correlation function}

In this section, we discuss scattering from the perspective of a continuous electron density function, $\rho(\boldsymbol{x})$, which we can mathematically construct considering the geometry of the material structure. This method rose to prominence from the work of Debye, Anderson, Brumberger, and Bueche, and will be further referred to as the Debye model. ${ }^{99,100}$ Consider a solid with density of electrons $\rho(\boldsymbol{x})$ in a given volume $V$ :

$$
\rho(\boldsymbol{x})=\rho_{0}+\eta(\boldsymbol{x})
$$

where $\rho_{0}$ is the average electron density and $\eta(\boldsymbol{x})$ represents the fluctuations around $\rho_{0}$. In the discrete description above, eqn (3) includes values for the positions of individual atoms and their atomic form factor $f_{j}$. In the continuous description, we replace the form factors $f_{j}$ with $\rho(\boldsymbol{x})$ (eqn (4)), and the scattered intensity may be written as:

$$
I(\boldsymbol{q})=I_{0} \int_{V} \int_{V} \rho\left(\boldsymbol{x}_{\boldsymbol{j}}\right) \rho\left(\boldsymbol{x}_{\boldsymbol{k}}\right) \mathrm{e}^{-i \boldsymbol{q} \cdot\left(\boldsymbol{x}_{\boldsymbol{k}}-\boldsymbol{x}_{\boldsymbol{j}}\right)} \mathrm{d}^{3} \boldsymbol{x}_{\boldsymbol{j}} \mathrm{d}^{3} \boldsymbol{x}_{\boldsymbol{k}}
$$

where $\rho\left(\boldsymbol{x}_{\boldsymbol{j}}\right)$ and $\rho\left(\boldsymbol{x}_{\boldsymbol{k}}\right)$ are the electron densities at points $\boldsymbol{x}_{\boldsymbol{j}}$ and $\boldsymbol{x}_{\boldsymbol{k}}$, respectively. The intensity can be rewritten as a function of the electron density fluctuations $\eta(\boldsymbol{x})$ in the material (see Appendix):

$$
I(\boldsymbol{q})=I_{0} \int_{V} \int_{V} \eta(\boldsymbol{x}) \eta(\boldsymbol{x}+\boldsymbol{r}) \mathrm{e}^{-i \boldsymbol{q} \cdot \boldsymbol{r}} \mathrm{d}^{3} \boldsymbol{x} \mathrm{d}^{3} \boldsymbol{r}
$$

In eqn (6), we have substituted $\boldsymbol{r}=\boldsymbol{x}_{\boldsymbol{k}}-\boldsymbol{x}_{\boldsymbol{j}}$, the distance between scatterers, and removed the subscript on $\boldsymbol{x}_{\boldsymbol{j}}$. Debye defines the correlation function $\gamma(\boldsymbol{r})$ as: ${ }^{99}$

$$
\int_{V} \eta(\boldsymbol{x}) \eta(\boldsymbol{x}+\boldsymbol{r}) \mathrm{d}^{3} \boldsymbol{x}=\overline{\eta^{2}} V \gamma(\boldsymbol{r})
$$

The mean square fluctuation, $\overline{\eta^{2}}$, is defined in the Appendix. In an isotropic system, $\gamma(\boldsymbol{r})$ depends only on the magnitude $|\boldsymbol{r}|=r$, and corresponds to the probability of two endpoints of a rod with length $r$ being within the same type of matter. For example, it is the probability of being in the solid phase of a NP material after moving a radius $r$ away from any random point in the NP material's solid phase. ${ }^{101}$ Thus, the correlation function will tend to 1 (having been normalized by $\overline{\eta^{2}}$ and $V$ ) for $r \rightarrow 0$ (short distances correlate very well), and 0 for $r \rightarrow \infty$ (long distances do not correlate). After some manipulation and assuming isotropy (using the average value of $\mathrm{e}^{-i \boldsymbol{q} \cdot \boldsymbol{r}}=\sin (q r) /$ $(q r)$, replacing the vectors $\boldsymbol{q}$ and $\boldsymbol{r}$ with their magnitudes), the intensity integral can be written as: ${ }^{100}$

$$
I(q)=I_{0} \overline{\eta^{2}} V \int_{0}^{\infty} \gamma(r) 4 \pi r^{2} \frac{\sin (q r)}{q r} \mathrm{~d} r
$$

Several system parameters may be extracted from the correlation function, whose functional form is developed from arguments based on some knowledge of the system. Debye et al. used a 
'square wave' (for details, see ref. 100) model of electron density to construct the correlation function $\left(\gamma_{\mathrm{D}}(r)\right.$, eqn (9)), and with it derived the surface-area-to-volume ratio $(S / V$, eqn (10)) in an inhomogeneous and porous medium with void fraction $\phi:{ }^{100}$

$$
\begin{gathered}
\gamma_{\mathrm{D}}(r)=\mathrm{e}^{\left(-\frac{r}{\xi}\right)} \\
\frac{S}{V}=-4 \phi(1-\phi) \gamma_{\mathrm{D}}^{\prime}(0) \rightarrow \frac{S}{V}=\frac{4 \phi(1-\phi)}{\xi}
\end{gathered}
$$

In eqn (9) and (10), $\xi$ is the mean width of the Debye correlation function and is called the correlation length, a characteristic length scale which describes how fast electron density fluctuation correlations decay spatially. $\gamma_{\mathrm{D}}{ }^{\prime}(0)$ represents the derivative of the correlation function at $r=0$. Thus, with knowledge of the functional form of the correlation function and the void fraction $\phi,{ }^{100}$ the surface-area-to-volume ratio can be obtained. Note that the surface-area-to-volume ratio is a function of the derivative of $\gamma_{\mathrm{D}}(r)$ when $r \rightarrow 0$, as this region describes correlations at smaller length scales (interfaces).

The specific surface area may also be calculated from the region over which the intensity decays as $q^{-4}$ (note, this power law is not always -4 , as will be discussed in Section 3.4) without any prior knowledge of $\gamma(r)$ by analyzing scattering data in absolute units, or by normalizing with the Porod invariant (see Appendix). The latter method has been described in numerous articles including Dotzler et al. who used it to calculate the specific surface area of nanoporous gold (NP-Au) during dealloying in nitric acid..$^{90,101,102}$ The result was independently discovered by Guinier and Debye's contemporary, Porod, and is thus named the Porod regime. ${ }^{90,100}$ For clarity, this method has been detailed in the Appendix.

After performing the Fourier transform of $\gamma_{\mathrm{D}}(r)$, the resultant intensity function is:

$$
I_{\mathrm{D}}(q)=\frac{I_{0} 8 \pi \xi^{3} \overline{\eta^{2}} V}{\left(1+q^{2} \xi^{2}\right)^{2}}
$$

This function does not contain a Bragg-like peak (Fig. 3a), and thus does not map onto the structure of NP materials which have some characteristic length scale not captured by $I_{\mathrm{D}}$.

\subsection{Models for nanoporous materials with a strong scattering peak}

In the late 1980s and early 1990s, there were many different approaches to determine the origin of a peak in the scattering data of bicontinuous microemulsions (oil and water mixtures). ${ }^{103-113}$ Depending on the temperature and composition of the mixture, the oil and water arrange into different microstructural phases. One such phase is bicontinuous, implying that if one were to start moving within the water phase, they could traverse the entire water network without crossing into the oil phase. While they look disordered and random, bicontinuous materials have a characteristic and quasi-repetitive length scale which gives rise to a Bragg-like scattering peak in the small-angle scattering regime. ${ }^{103-113}$ Many NP material classes exhibit such a bicontinuous structure, ${ }^{85,114-118}$ opening up an opportunity to study these systems with X-ray scattering. One such class is NP metals, typically synthesized by an (electro)chemical etching process known as dealloying, in which the sacrificial component of an alloy is selectively removed to form a bicontinuous network of struts (ligaments) and channels (pores), both with characteristic diameter in the nanoscale range. ${ }^{87,119-127}$ Advances in tomography techniques have enabled 3D reconstruction of several NP metals; these findings indicate that the ligament and pore structures are inverse of one another and are morphologically and topologically equivalent, and thus exhibit the so-called bicontinuous structure. ${ }^{128-130}$ Computation of NP metal structures with Kinetic Monte Carlo, molecular dynamics, and phase field methods yield similar results. ${ }^{85,123,125-127,131-135}$ These bicontinuous networks (both NP metals and microemulsions), have been modeled as spinodally decomposed structures. ${ }^{85,105,113,119,136-138}$ In this section we focus on this type of microstructure and how SAXS can aid in determining this microstructure.

3.3.1 Cahn spinodal decomposition. The spinodal decomposition model stems from the work of J. W. Cahn and J. E. Hilliard, who developed a model for the free energy function for a non-uniform system in the late 1950 s. ${ }^{139}$ In several subsequent articles, Cahn describes spinodal decomposition of isotropic systems in terms of local concentration, $c(\boldsymbol{q}, \boldsymbol{r} ; \boldsymbol{t})$, and its gradients (see Appendix). ${ }^{120,140-142}$ In essence, the spinodal concentration $c(\boldsymbol{q}, \boldsymbol{r} ; \boldsymbol{t})$ is found by implementing the Cahn-Hilliard free energy expansion into Fick's diffusion equations and solving them in terms of Fourier components, leading to a superposition of waves:

$$
c-c_{0}=\sum_{\boldsymbol{q}} \mathrm{e}^{R(q) t}(A(\boldsymbol{q}) \cos (\boldsymbol{q} \cdot \boldsymbol{r})+B(\boldsymbol{q}) \sin (\boldsymbol{q} \cdot \boldsymbol{r}))
$$

Where $R(q)$ is the amplification factor (defined in Cahn), ${ }^{120}$ and $\boldsymbol{q}$ is the wavevector of the Fourier components with magnitude $q$. By assuming the only important Fourier component is $\boldsymbol{q}_{0}$, that which maximizes $R(q), \Sigma_{\boldsymbol{q}}$ can be replaced by $\Sigma_{\boldsymbol{q}_{0}}$ in eqn (12). In this case, $c$ becomes a superposition of waves that all have the same magnitude wavevector, but with random orientation, amplitude, and phase. ${ }^{120}$ Cahn computed several binarized 2D level cuts of this so-called stochastic standing wave in one of the first computer simulations of material structure, ${ }^{120}$ predicting the bicontinuous two-phase structure of characteristic size denoted by the wavelength of the maximizing wavevector $q_{0}$, i.e. $\frac{2 \pi}{\lambda_{0}}$.

3.3.2 Leveled-wave model. Recently, Soyarslan et al. provided evidence for the applicability of Cahn's model to NP metals by mapping it to their mechanical behavior and specific surface area. $^{122}$ We use their notation for clarity. Cahn's stochastic standing wave equation (eqn (12)) is restructured into cosine waves with random orientation and phase:

$$
f(\boldsymbol{x})=\sqrt{\frac{2}{N}} \sum_{i=1}^{N} \cos \left(\boldsymbol{q}_{i} \cdot \boldsymbol{x}+\varphi_{i}\right)
$$

where $N$ is the total number of waves used, $\boldsymbol{q}_{\boldsymbol{i}}$ are the random directions distributed on a sphere with radius $q_{0}$ (i.e. the wavevectors have equivalent magnitude), and $\varphi_{i}$ are random phases. Once $N$ waves are generated $\left(10^{4}\right.$ waves are used, as 


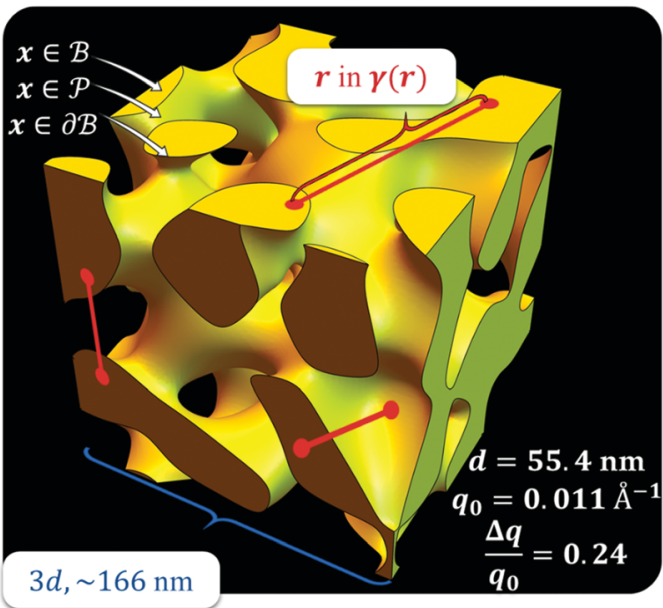

Fig. 2 Example of a 3D structure generated by the leveled-waved model found in Berk ${ }^{111,112}$ and Soyarslan et al. ${ }^{122}$ using one wavelength, $\boldsymbol{q}_{0}$ determined by fitting the dataset in Section 4.

mentioned in Soyarslan et al., to ensure isotropy in the simulated structure), ${ }^{122}$ points in real space are assigned as part of the bulk or void by thresholding $f(\boldsymbol{x})$ with a value $\xi$ (not to be confused with the correlation length):

$$
\boldsymbol{x} \in \mathcal{B} \text { if } f(\boldsymbol{x})<\xi, \quad \boldsymbol{x} \in \partial \mathcal{B} \text { if } f(\boldsymbol{x})=\xi, \quad \boldsymbol{x} \in \mathcal{P} \text { if } f(\boldsymbol{x})>\xi
$$

where $\mathcal{B}$ is the bulk (the metal), $\partial \mathcal{B}$ is the interface, and $\mathcal{P}$ is the pore. ${ }^{122}$ The threshold value is a function of the bulk fraction $\phi_{\mathcal{B}}, \xi=\sqrt{2} \operatorname{erf}^{-1}\left(2 \phi_{\mathcal{B}}-1\right)$. This thresholding process produces a leveled-wave, which can be computed to provide structural visualization for a scattering dataset. As a demonstration of this concept for one wavelength, we used Mathematica ${ }^{143}$ to compute one such structure, which is displayed in Fig. 2. Here we used fitted values (discussed later in Section 4) for ligamentligament distance to determine $q_{0}$.

3.3.3 Berk model. In two mathematically rigorous articles on the subject, N. F. Berk used the leveled-wave scheme discussed above to investigate the scattering properties of a three-phase system (such as oil/interface/water), which can be reduced to a two-phase system (such as ligament/pore in NP metals). ${ }^{111,112}$ The correlation function for $f(\boldsymbol{x})$ is the first spherical Bessel function: ${ }^{122}$

$$
\gamma_{\mathrm{LW}}(r)=\frac{\sin \left(q_{0} r\right)}{q_{0} r}=j_{0}\left(q_{0} r\right)
$$

The Fourier transform of eqn (14) predicts a very sharp peak at $q_{0}$, as the function used only one magnitude of $q$ (i.e. $q_{0}$ ), unlike the SAXS data shown in Fig. 3. Berk added Gaussian dispersion around $q_{0}$ and solved for the scattering intensity analytically: ${ }^{111,112}$

$$
I_{\mathrm{B}}(q)=\frac{1}{q} \frac{\left(\frac{\pi}{2}\right)^{\frac{3}{2}}}{2 q_{0}(\Delta q)}\left[\exp \left(-\frac{\left(q-q_{0}\right)^{2}}{2(\Delta q)^{2}}\right)-\exp \left(-\frac{\left(q+q_{0}\right)^{2}}{2(\Delta q)^{2}}\right)\right]
$$

for which the probability density for the wavevector $q$ is a Gaussian distribution centered around $q_{0}$ with variance $(\Delta q)^{2} \cdot{ }^{112}$ This function

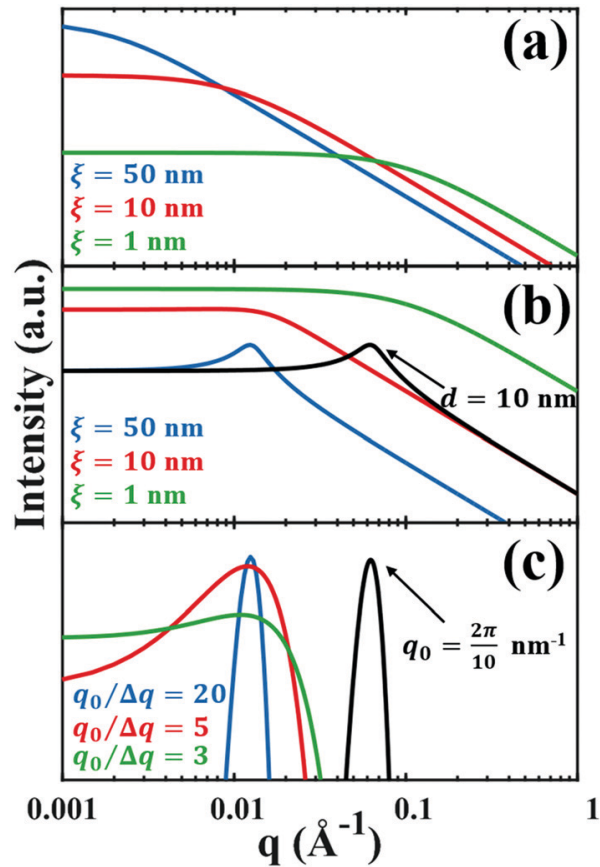

Fig. 3 Comparison of the (a) Debye, (b) Teubner-Strey, and (c) Berk models and variation in their key parameters. In the Debye model, altering the correlation length shifts the curve, but it maintains its shape. In the TS (b) and Berk (c) models, the curves center around a value of $q$ corresponding to the characteristic size (i.e. $\boldsymbol{d}$ or $\boldsymbol{q}_{0}$ ). Here, the colored lines represent a constant characteristic size and we vary the correlation length (in b), or the ratio $\boldsymbol{q}_{0} / \Delta \boldsymbol{q}$ (in c), to alter the distribution around that $q$-value. In the black curves, we have changed the characteristic size while maintaining the distribution in the blue curve.

has been reduced from its original form (eqn (11) in Berk), where here we are assuming equal pore and bulk volume percentages $\left(\phi_{\mathcal{B}}=\phi_{\mathcal{P}}=0.5\right)$. Since this intensity function is derived based on a correlation function expansion that does not converge quickly as $r \rightarrow 0$, the asymptotic curve at higher values of $q$ (in the Porod regime) cannot be well matched with any finite number of expansion terms. ${ }^{111}$ Eqn (15) has been plotted in Fig. 3c, which shows how this function changes when the both distribution $(\Delta q)$ and the characteristic spacing $\left(q_{0}\right)$ are varied. We can clearly see that this intensity distribution does not match the high- $q$ tail of the NP-Au scattering spectrum in Fig. 5 .

3.3.4 Teubner-Strey (TS) model. In another attempt to determine the origin of the scattering peak in microemulsions, Teubner and Strey developed a free energy expansion in terms of an order parameter, $\psi$, and its gradients, $\nabla \psi:^{113}$

$$
\begin{gathered}
f\left(\psi, \nabla \psi, \nabla^{2} \psi, \ldots\right)=a_{0}+a_{1} \psi+a_{2} \psi^{2}+a_{3} \psi^{3}+\ldots+c_{1}(\nabla \psi)^{2} \\
+c_{2}\left(\nabla^{2} \psi\right)^{2}+\ldots
\end{gathered}
$$

where $f$ is the local free energy per molecule. The order parameter $\psi$ in the case of microemulsions is the water-oil ratio, and all coefficients except for $a_{2}>0, c_{1}<0$, and $c_{2}>0$ were taken to be zero. Choosing these coefficient values was a system-based decision: $a_{0}$ and $a_{1}$ are zero by symmetry, terms higher than $a_{2}$ were ignored as the fluctuations are small, $c_{1}$ was chosen to be negative as there is negative microscopic 
surface tension in microemulsions and considering this, $c_{2}$ must be positive to provide phase stability. ${ }^{113}$ This methodology is known as Landau-Ginzberg phenomenological theory, and we refer the reader to Chapter 3 of Kardar's Statistical Physics of Fields, which connects it to scattering. ${ }^{144}$ Upon Fourier transformation of the fluctuations of $\psi$, one can extract a correlation function and a scattering intensity relationship with two length scales, $d$ and $\xi$ :

$$
\begin{aligned}
\gamma_{\mathrm{TS}}(r) & =\mathrm{e}^{-\left(\frac{r}{\xi}\right)} j_{0}\left(\frac{2 \pi r}{d}\right) \\
I_{\mathrm{TS}}(q) & =\frac{\frac{8 \pi}{\xi} \eta^{2} c_{2} V}{a_{2}+c_{1} q^{2}+c_{2} q^{4}}
\end{aligned}
$$

where fit parameters $a_{2}$ and $c_{1,2}$ are functions of $d$ and $\xi$. Here, $d$ is a characteristic domain size (periodicity) which captures local order and the peak in the intensity distribution; $\xi$ is the correlation length which, as mentioned previously, describes how fast electron density fluctuation correlations decay spatially. We have plotted eqn (17) in Fig. 3b to demonstrate how the intensity changes with parameters $d$ (represented by the black curve) and $\xi$ (represented by the colored curve). As shown in Fig. 3, The Teubner-Strey correlation function combines those of Debye, $\mathrm{e}^{(-r / \xi)}$, and Berk, $j_{0}\left(q_{0} r\right)$, so that $I_{\mathrm{TS}}$ captures both the peak and a characteristic $q^{-4}$ Porod regime. This model has been used extensively in the microemulsion literature due to its satisfactory evaluation of characteristic size and ease of model fitting. ${ }^{103,106,145,146}$ However, it misses an important intermediate regime after the peak and before the Porod regime, which is present in some bicontinuous microemulsions and is a consequence of another length scale in the scattered volume. The following model adds a third length scale, $\delta$, to capture this intermediate regime, which appears in the data as a shoulder and corresponds to interfacial roughness, or the correlations in the wrinkles at the surface.

3.3.5 Choi and Chen modified leveled-wave model. The Debye, Berk, and Teubner-Strey models do not adequately describe the entire scattering intensity curve of NP-Au, as will be discussed further in Section 4. To deal with this discrepancy, Choi and Chen introduced a third length scale that modifies the Berk model - an interfacial length $\delta .{ }^{145}$ Their model replaces the single wavevector magnitude, $q_{0}$, for $\boldsymbol{q}_{i}$ in eqn (13), with a distribution of magnitudes, $f(q)$, called a spectral density function. This distribution can take on any form as long as it is normalizable, i.e. $\int_{0}^{\infty} f(q) \mathrm{d} q=1$, however the choice should have some relevance to the scattering data. ${ }^{111}$ Choi and Chen have used an inverse 8thorder polynomial with three length scales $\left(a, b\right.$, and $c$ ) for $f(q):{ }^{145}$

$$
f(q)=\frac{b c\left[a^{2}+(b+c)^{2}\right]^{2} /(b+c) \pi^{2}}{\left(q^{2}+c^{2}\right)^{2}\left[q^{4}+2\left(b^{2}-a^{2}\right) q^{2}+\left(a^{2}+b^{2}\right)^{2}\right]}
$$

where $a=\frac{2 \pi}{d}, b=\frac{1}{\xi}$, and $c=\frac{1}{\delta}$. It was shown by Teubner that the Gaussian curvature $\langle K\rangle$ and the square mean curvature $\left\langle H^{2}\right\rangle$ are functions of the 2nd and 4th moments of $f(q),\left\langle q^{2}\right\rangle$ and $\left\langle q^{4}\right\rangle$, respectively. ${ }^{104}$ This high degree polynomial was chosen in order to capture the 4 th moment, whereas in the other cases (Debye, Berk, and TS models), only odd moments exist, or the 4th moment diverges. ${ }^{145}$ Curvature analysis in this model is therefore more robust than in the aforementioned models. One can obtain these parameters by fitting a form of $f(q)$ to an experimental correlation function $\gamma_{\mathrm{E}}(r)$ using the steps outlined in the Appendix.

We will study the pattern of NP-Au in more details in Section 4, during which the Choi and Chen model will be used, and we will provide an example of interfacial shape distribution (ISD) analysis to share its usefulness as a tool for quantitative analysis of ISD evolution and evaluation of self-similarity in coarsening. For the remainder of Section 3 (namely Section 3.4 below), we will discuss scattering from porous materials that do not give rise to a Bragg-like peak.

\subsection{Models for nanoporous materials without a strong scattering peak}

While NP materials with a well-defined and quasi-repetitive characteristic length over a long range give rise to a strong, Bragg-like scattering peak as mentioned in the previous sections, other NP materials do not. Instead of a peak, they exhibit distinct features in their scattering spectra. In particular, 'shoulders,' or plateaus, in the data indicate an important length scale that can be characterized by combining both the high- $q$ Porod-like powerlaw decay discussed in Section 3.2 and low- $q$ analysis put forward by Guinier. ${ }^{90}$ Guinier approximated the scattering at very low angles to decay exponentially in what is sometimes referred to as Guinier's law: ${ }^{90}$

$$
I(q) \sim \mathrm{e}^{-\frac{q^{2} R_{\mathrm{G}}^{2}}{3}}
$$

introducing the concept of the radius of gyration $\left(R_{\mathrm{G}}\right)$ in scattering. In the context of scattering, the radius of gyration of an object (of arbitrary shape) is the root-mean-squared distance from its electronic center of mass. This quantity can be obtained from the slope of a linear fit of $\ln (I(q)) v s . q^{2}$ in the so-called Guinier regime $\left(R_{\mathrm{G}} \times q<1.3\right.$, for spherical objects $\left.{ }^{90}\right)$. The relationship between the radius of gyration and some real physical length depends on the shape of the object in question. For a spherical particle, for example, $R_{\mathrm{G}}{ }^{2}=\frac{3}{5} R_{\mathrm{p}}{ }^{2}$, where $R_{\mathrm{p}}$ is the radius of the particle. ${ }^{96}$ Feigin and Svergun have tabulated several of these relationships for simple shapes, ${ }^{147}$ and this relatively simple analysis is performed frequently in X-ray and neutron scattering data. ${ }^{45,148-154}$ In a seminal paper on carbidederived carbon, Gogotsi et al. fit scattering data to show that there is a very narrow distribution of pores which can be controlled well by synthesis temperature. ${ }^{45}$ They used an analysis put forward by Kyutt et al. which determines pore size by a series of $R_{\mathrm{G}}$ fits of the SAXS data. ${ }^{155-157}$

$R_{\mathrm{G}}$ was derived for particles of various shapes which are separated from each other in some solution, ${ }^{90}$ however many NP materials consist of grains comprised of nanoscopic subunits, often with a distribution of sizes, which are not widely separated. 
Such a material will scatter across those multiple length scales, producing overlapping spectra which can be difficult to interpret. For example, one may find a Guinier shoulder between two power-law decay regimes. Fitting this type of curve has been the subject of numerous models, as one would be hard-pressed to fit with any of those previously mentioned. Beaucage addressed the issue in a unified exponential/power-law model which, by way of local fitting in important regions (i.e. around the Guinier shoulder), one may determine physical parameters on these different length scales. ${ }^{151}$ Hammouda developed on the unified Beaucage model by adding continuity equations on some of the fitted parameters to eliminate discontinuities in the fits. ${ }^{150}$ Spalla et al. developed a model for two distinct power-law regimes which offers a way to extract physical parameters (specific surface area, pore radius, porosity) if the exact chemical composition of the sample is known. Using the Hammouda and Spalla models, Panduro et al. ${ }^{158}$ have studied the SAXS patterns of $\mathrm{CeO}_{3}$ particles of average radius $2.3 \mu \mathrm{m}$, which contain two distributions of pores (micro- and nanoscale). Using USAXS, they resolve the different pore sizes as separate entities with their own physical parameters (e.g. average size).

There are also cases where the Porod power-law decay deviates from $q^{-4}$ as discussed in Section 3.2. This deviation of $\alpha$ in $I(q) \sim q^{-\alpha}$ is controlled by the scatterer's fractal dimension, a notable concept in the scattering literature ${ }^{96,154,158-163}$ which is beyond the scope of this focus article. Martin and Hurd discuss the subject at length in an article on the subject. ${ }^{164}$ Briefly, the high- $q$ decay rate, $\alpha$, can be thought of as describing a particle's surface by its smoothness. For example, if $\alpha=4$, the surface is smooth, and when $\alpha=3$, the surface is rough. ${ }^{150}$ We also note that if $\alpha<3$, the scatterer is a mass fractal, and if $3<\alpha<4$, it is a surface fractal. ${ }^{150,164}$ Both the unified Beaucage model and Hammouda's extension can capture the deviation, and was part of the analysis in Panduro et al. ${ }^{158}$

Fig. 4 shows two different NP metal scatterers synthesized in our laboratory, namely nanoporous antimony (NP-Sb) and nanoporous copper (NP-Cu), which do not give rise to strong, Bragg-like scattering. The NP-Sb and NP-Cu were made by selective removal of sacrificial $\mathrm{Mn}$ from $\mathrm{Mn}$-rich $\mathrm{Sb}-\mathrm{Mn}$ and $\mathrm{Cu}-\mathrm{Mn}$ alloys, respectively (details of the synthesis are beyond

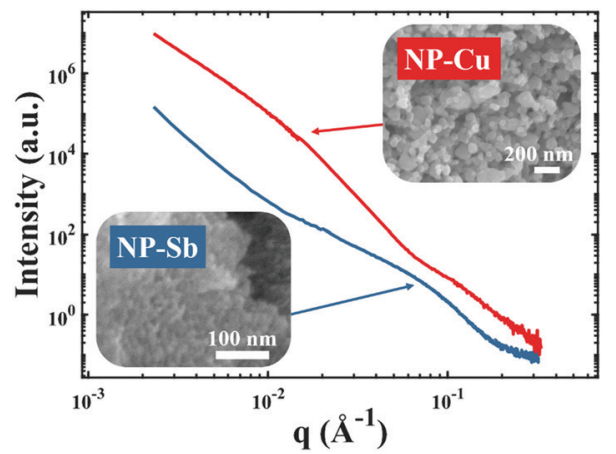

Fig. 4 Nanoporous materials which do not give rise to a strong, Bragglike scattering peak. Scattering patterns of NP-Cu (red curve) and NP-Sb (blue curve) and corresponding scanning electron micrographs. the scope of this article). We have attempted to use the various models mentioned in this section to fit these data, but by comparing to the SEM micrographs shown in the insets, we note that the models yield unrealistic parameters for characteristic size. Development of new models to fit these data are beyond the scope of this focus article, but will be the subject of a follow-up work.

\section{Case study for NP-Au}

The most common and practical way to tune the structural size of a NP metal is by thermal treatment, which has been the subject of numerous studies using various techniques, including $\mathrm{X}$-ray tomography, ${ }^{129,165}$ Laue diffraction, ${ }^{129}$ electron microscopy, ${ }^{76,166-170}$ positron annihilation spectroscopy, ${ }^{168}$ and thermal conductivity measurements. ${ }^{171}$ Kinetic Monte Carlo (KMC) simulations indicate that diffusion-controlled Rayleigh instabilities cause coarsening, where a reduction in topological genus drives growth of the framework. ${ }^{129,132}$ An X-ray tomography study by Chen-Wiegart et al. indicated that the scaled surface curvature and surface orientation evolves during coarsening, indicating it is non-self-similar. The self-similarity classification requires that the topology and morphology do not evolve temporally when scaled under a time-dependent quantity like surface area. ${ }^{129}$ More recent work by Lilleodden and co-workers have revealed that the coarsening process is nearly self-similar; ${ }^{172,173}$ however, the full picture of the coarsening process remains open for debate. ${ }^{174}$

In this section, we fit the models discussed in Section 3.3 to real scattering data of a $\sim 5 \mu \mathrm{m}$-thick film of NP-Au made by free corrosion of a $\mathrm{Au}_{35} \mathrm{Ag}_{65}$ leaf (custom order, Norris Blattgoldfabrik) in $15 \mathrm{M} \mathrm{HNO}_{3}$ for 30 minutes (gold curve in Fig. 5). These scattering data were acquired at the University of Pennsylvania's Dual Source and Environmental X-ray Scattering (DEXS) facility. The DEXS facility is home to a scattering instrument, equipped to probe length scales from $1 \AA$ to $570 \mathrm{~nm}$ by utilizing variable sample-to-detector distances (up to $6.4 \mathrm{~m}$ ), and both copper and molybdenum X-ray sources. The data in Fig. 5 combines two collections at different sample-to-detector distances to encompass a larger $q$ range, and intensity was calibrated to absolute units $\left(\mathrm{cm}^{-1}\right)$ with a glassy carbon standard. ${ }^{175}$

For all models shown in Fig. 5a, we have chosen equivalent bulk and pore fractions $(\phi=\phi=0.5)$, the isometric case, to compare with the Choi and Chen model. The values for volumetric and mass specific surface, $S_{\mathrm{V}}$ and $S_{\mathrm{m}}$, respectively, will decrease if the bulk fraction is changed, but not by orders of magnitude. Fit parameters and specific surface area calculations have been summarized in Table 1 . The ligament-ligament spacing, $d$, fitted by the Berk and Teubner-Strey models are nearly the same, $\sim 55 \mathrm{~nm}$. This characteristic distance in real space was evaluated by taking manual measurements of the SEM image in Fig. 5b, where 30 measurements of 'obvious' adjacent ligament centers (red lines in Fig. 5b are examples) yield a spacing of $\sim 62 \mathrm{~nm}$. To convert between $S_{\mathrm{V}}$ and $S_{\mathrm{m}}$, the effective density of NP-Au was chosen to be $0.65 \times 19.3 \mathrm{~g} \mathrm{~cm}^{-3}$, which takes into account its porosity when the 65 at\% sacrificial $\mathrm{Ag}$ content has been removed during free corrosion. 


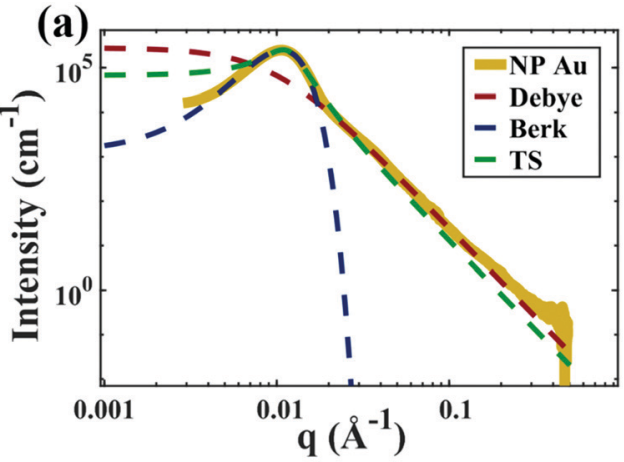

(b)

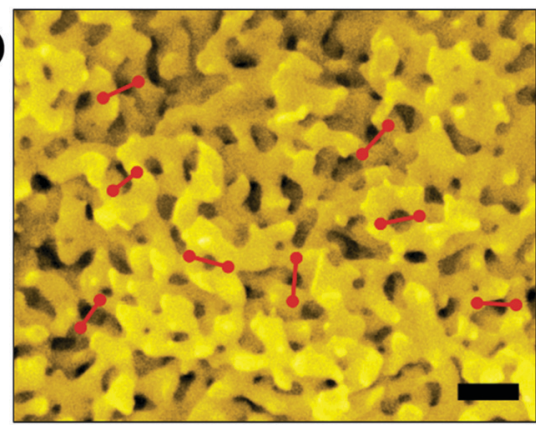

Fig. 5 (a) SEM and (b) SAXS data from a $\sim 5 \mu \mathrm{m}$-thick film of NP-Au dealloyed by free corrosion in concentrated $\mathrm{HNO}_{3}$ for 30 minutes (scale bar is $100 \mathrm{~nm}$ ). The peak in the scattering data comes from the characteristic ligament-ligament spacing (red lines on the SEM), and we model the data with the $\boldsymbol{I}(\boldsymbol{q})$ functions discussed in Section 3.3.

Table 1 Fitted parameters for all four models used to evaluate the SAXS data

\begin{tabular}{|c|c|c|c|c|}
\hline \multirow[b]{2}{*}{ Model } & \multicolumn{2}{|c|}{$\underline{\text { Fitted parameters }}$} & \multicolumn{2}{|c|}{ Predicted values } \\
\hline & $\Delta q / q_{0}$ & $d(\mathrm{~nm})$ & $S_{\mathrm{v}}\left(\mathrm{m}^{2} \mathrm{~cm}^{-3}\right)$ & $S_{\mathrm{m}}\left(\mathrm{m}^{2} \mathrm{~g}^{-1}\right)$ \\
\hline \multirow[t]{2}{*}{ Berk } & 0.24 & 55.3 & 66.1 & 3.4 \\
\hline & \multicolumn{2}{|c|}{ Fitted parameters } & \multicolumn{2}{|c|}{$\underline{\text { Predicted values }}$} \\
\hline Model & $\xi(\mathrm{nm})$ & $d(\mathrm{~nm})$ & $S_{\mathrm{v}}\left(\mathrm{m}^{2} \mathrm{~cm}^{-3}\right)$ & $S_{\mathrm{m}}\left(\mathrm{m}^{2} \mathrm{~g}^{-1}\right)$ \\
\hline Debye & 10.3 & - & 149.3 & 7.7 \\
\hline Teubner-Strey & 31.9 & 55.5 & 48.3 & 2.5 \\
\hline
\end{tabular}

Of the models fitted in Fig. 5a, the Teubner-Strey model is clearly the most descriptive of the real scattering data. However, it fails to capture the intermediate shoulder in the curve after the peak and through its transition into the high- $q$ Porod scattering regime $\left(I(q) \sim q^{-4}\right)$. This shoulder corresponds to another characteristic length scale in the system - we model this third length scale with the Choi and Chen model parameter $\delta$ (surface roughness parameter), generate structures that look qualitatively similar to NP-Au, and evaluate the mean and Gaussian curvatures of these structures (Fig. 6). The fitted $\delta$ value was on the order of $3 \mathrm{~nm}$. One can immediately distinguish the structure in Fig. 6a (Choi and Chen spectral distribution, eqn (18)) from the structure in Fig. 2, which uses a single magnitude of $q$, by a few distinct features. Namely, there are 'capped' regions, and 'necked' regions in this simulated structure. Fig. $6 \mathrm{~b}$ and $\mathrm{c}$ show that the curvature of much of the structure is nearly zero, however, (a)

(b)
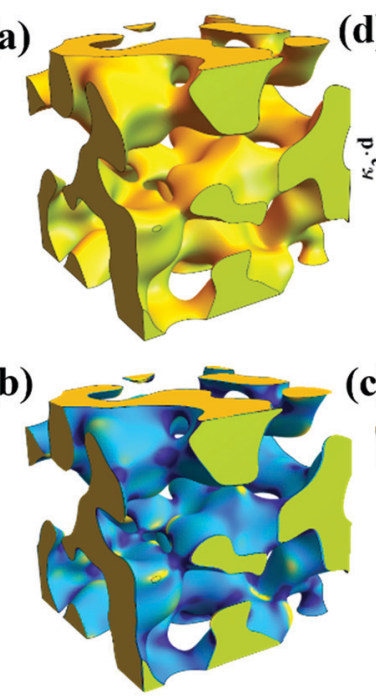

$K_{G}=\kappa_{2} \kappa_{1}$

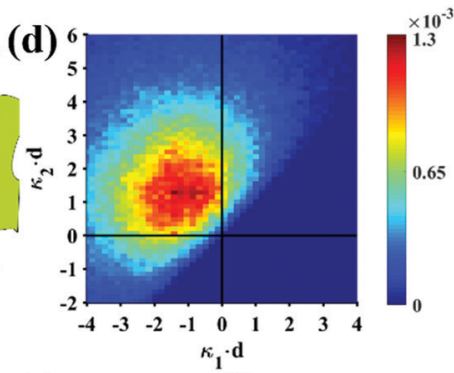

(c)

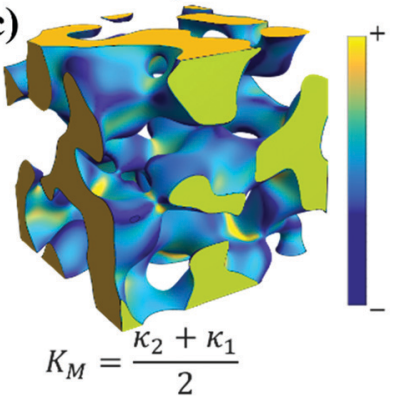

Fig. 6 Structure generation and ISD analysis using the Choi and Chen model fits to real experimental data from Fig. 3. (a) 3D structure generated from fitted Choi and Chen model, (b and c) Gaussian and mean curvature maps of (a), using the convention that convex regions have negative mean curvature. (d) Interfacial shape distribution of the generated structure.

the necked and capped regions have non-zero curvature. These regions are integral to the coarsening behavior, as they represent the parts of the structure involved in the Rayleigh instabilities that reduce the material's topological genus. ${ }^{127,165,172,174}$

\section{Conclusion}

In this focus article, we have introduced SAXS as an important tool to characterize the structure of NP materials. To bring unfamiliar and interested investigators up to speed, we discuss the geometry of a scattering experiment and develop SAXS theory from fundamentals. We explore a variety of models to fit our data, starting from the Debye model, and adding multiple length scales in the Berk, Teubner-Strey, and Choi \& Chen models to capture the significant features of the scattering intensity of NP materials with a strong scattering peak. We successfully apply these models to the scattering pattern of NP-Au in a case study, and put SAXS forward as a method to investigate the morphological characteristics of NP materials by linking structural similarities to bicontinuous microemulsions. In addition, we have discussed the scattering patterns of nanoporous materials without strong scattering peak. It is found that the models investigated in this work do not yield realistic material parameters for specific nanoporous copper and nanoporous antimony morphologies, however. A follow-up work will investigate appropriate models for these morphologies.

\section{Conflicts of interest}

There are no conflicts to declare. 


\section{A. Appendix}

\section{A.1 Atomic form factor $f_{j}$}

In Section 3, we described the atomic form factor as the scattering ability of the $j$ th atom. The atomic form factor takes into consideration the probability distribution of electrons in an atom, which can be described by the square of its wavefunction, $|\psi(\boldsymbol{r})|^{2}=\rho_{\mathrm{a}}(\boldsymbol{r})$. Its mathematical form is similar to that of the continuous electron distribution discussed in Section 3.2 (i.e., a Fourier transform):

$$
f_{j}=\int \rho_{\mathrm{a}}(\boldsymbol{r}) \mathrm{e}^{i \boldsymbol{q} \cdot \boldsymbol{r}} \mathrm{d}^{3} \boldsymbol{r}
$$

Thus, it is a function of the scattered angle, the radiation's wavelength, and the distribution of electrons in the atom. All atomic form factors decrease from the atomic number $Z$ at $q=0$ to 0 at high $q .^{176,177}$ NIST $^{178}$ and the International Tables for Crystallography ${ }^{179}$ have useful tables and calculators for the form factors.

\section{A.2 Detailed derivation of scattering from a continuous charge distribution}

The following derivation stems from two seminal papers of Debye and co-workers, ${ }^{99,100}$ and can also be found in Chapter 2 of Small-Angle Scattering of X-rays. ${ }^{90}$ As defined in Section 3.2,

$$
\rho(\boldsymbol{x})=\rho_{0}+\eta(\boldsymbol{x})
$$

Which can be rewritten as follows:

$$
\eta(\boldsymbol{x})=\rho(\boldsymbol{x})-\rho_{0}
$$

The average value of the fluctuation is zero, meaning that:

$$
\int_{V} \eta(\boldsymbol{x}) \mathrm{d}^{3} x=\int_{V}\left[\rho(\boldsymbol{x})-\rho_{0}\right] \mathrm{d}^{3} \boldsymbol{x}=0
$$

In Section 3.2 we have left out the steps between eqn (5) and (6), so we have included them here for convenience. Substituting eqn (4) into equation eqn (5) yields:

$$
I(\boldsymbol{q})=I_{0} \int_{V} \int_{V}\left[\rho_{0}+\eta\left(\boldsymbol{x}_{\boldsymbol{j}}\right)\right]^{*}\left[\rho_{0}+\eta\left(\boldsymbol{x}_{\boldsymbol{k}}\right)\right] \mathrm{e}^{-i \boldsymbol{q} \cdot\left(\boldsymbol{x}_{\boldsymbol{k}}-\boldsymbol{x}_{\boldsymbol{j}}\right)} \mathrm{d}^{3} \boldsymbol{x}_{\boldsymbol{j}} \mathrm{d}^{3} \boldsymbol{x}_{\boldsymbol{k}}
$$

We can break up this integral into four parts, including the principal and cross terms. We can ignore three of the terms, ${ }^{90}$ and we are left with only one term will remain as given in eqn (25):

$$
I(\boldsymbol{q})=I_{0} \int_{V} \int_{V}\left[\eta\left(\boldsymbol{x}_{j}\right)\right]^{*}\left[\eta\left(\boldsymbol{x}_{\boldsymbol{k}}\right)\right] \mathrm{e}^{-i \boldsymbol{q} \cdot\left(\boldsymbol{x}_{\boldsymbol{k}}-\boldsymbol{x}_{\boldsymbol{j}}\right)} \mathrm{d}^{3} \boldsymbol{x}_{\boldsymbol{j}} \mathrm{d}^{3} \boldsymbol{x}_{\boldsymbol{k}}
$$

We substitute $\boldsymbol{r}=\boldsymbol{x}_{\boldsymbol{k}}-\boldsymbol{x}_{\boldsymbol{j}}$, and consider the $\boldsymbol{x}$ integral:

$$
\int_{V} \eta(\boldsymbol{x}) \eta(\boldsymbol{x}+\boldsymbol{r}) \mathrm{d}^{3} \boldsymbol{x}
$$

If $|\boldsymbol{r}|=0$, this integral goes to:

$$
\int_{V} \eta(\boldsymbol{x}) \eta(\boldsymbol{x}) \mathrm{d}^{3} \boldsymbol{x}=\overline{\eta^{2}} V
$$

defining the mean squared fluctuations of electronic density, $\overline{\eta^{2}}$ where $V$ represents the irradiated volume. This factor is used to normalize the correlation function $\gamma(\boldsymbol{r})$ to 1 .

\section{A.3 Inverting intensity, Porod invariant, and specific surface}

While we cannot obtain the spatial distribution of electrons from scattering data directly, we can extract the correlation function using the inverse Fourier transform:

$$
I_{0} V \overline{\eta^{2}} \gamma(r)=\frac{1}{2 \pi^{2}} \int_{0}^{\infty} I(q) q^{2} \frac{\sin (q r)}{q r} \mathrm{~d} q
$$

As stated in Section 3.2, the correlation function tends to 1 as $r \rightarrow 0$, and 0 as $r \rightarrow \infty$. We can use this fact to obtain the Porod invariant, $Q^{*}$, where we set $r=0$ in the Fourier inversion equation:

$$
2 \pi^{2} I_{0} V \overline{\eta^{2}}=\int_{0}^{\infty} I(q) q^{2} \mathrm{~d} q=Q^{*}
$$

The Porod invariant is named as such because it varies only with the mean squared fluctuations of electron density within the scattered volume, and not their spatial arrangement (i.e. their structure). ${ }^{101}$ In a two-phase system with electron densities $\rho_{1}$ and $\rho_{2}$, we can use the fact that the mean squared fluctuations $\overline{\eta^{2}}=\Phi_{1} \Phi_{2}\left(\rho_{2}-\rho_{1}\right)^{2}$ to associate $Q^{*}$ with physical properties of the scattered volume: ${ }^{101}$

$$
Q^{*}=\Phi_{1} \Phi_{2}\left(\rho_{2}-\rho_{1}\right)^{2} V I_{0} 2 \pi^{2}
$$

In the case of NP-Au scattering in vacuum, the contrast $\Delta \rho=\rho_{2}-\rho_{1}$ is simply equivalent to the electron density of $\mathrm{Au}$ $\left(\rho_{1}=0\right), \Phi_{1}$ is the void fraction, and $\Phi_{2}=\left(1-\Phi_{1}\right)$. When absolute intensity calibration is not possible, $Q^{*}$ can be used to normalize the data in order to extract specific surface area: ${ }^{96,102}$

$$
\frac{S}{V}=\frac{\pi \lim _{q \rightarrow \infty} I(q) q^{4}}{Q^{*}} \Phi_{1} \Phi_{2}
$$

In this way, we can determine the specific surface area without using absolute calibration by plotting the normalized $\frac{I(q)}{Q^{*}} q^{4}$ vs. $q$ and finding the constant value it tends to. It is important to note, however, that in a scattering experiment we do not have data for $q \rightarrow \infty$, thus absolute intensity will give a more accurate representation of the specific surface. If the intensity data is in absolute units, the invariant calculation is not needed, and the specific surface is:

$$
\frac{S}{V}=\frac{\lim _{q \rightarrow \infty} I(q) q^{4}}{2 \pi\left(\rho_{2}-\rho_{1}\right)^{2}}
$$

\section{A.4 Fitting Choi \& Chen model}

An experimental correlation function $\gamma_{\mathrm{E}}(r)$ (found by extrapolating and Fourier transforming the $I(q)$, we used SASview to accomplish this) ${ }^{180}$ can be fitted to the spectral density function $f(q)$ in eqn (18) by the following analytical relationship for an isometric $\left(\phi_{\mathrm{B}}=\phi_{\mathrm{P}}=0.5\right)$ 
two-phase system as shown in Berk, and Choi et al::111,145

$$
\gamma(r)=\frac{2}{\pi} \arcsin g(r)
$$

where $g(r)=\int_{0}^{\infty} f(q) j_{0}(q r) \mathrm{d} q$. Choi and Chen explicitly solve for $g(r)$ in their first article on the subject. ${ }^{146}$

\section{Acknowledgements}

The authors are thankful to Penn Engineering for the financial support through the PI startup and to the Vagelos Institute for Energy Science and Technology (VIEST) for the support through the 2019 VIEST Graduate Fellowship support. The authors acknowledge use of the Dual Source and Environmental X-ray Scattering facility operated by the Laboratory for Research on the Structure of Matter at the University of Pennsylvania (NSF MRSEC 17-20530). The equipment purchase was made possible by an NSF MRI grant (17-25969), an ARO DURIP grant (W911NF17-1-0282), and the University of Pennsylvania. This work was carried out in part at the Singh Center for Nanotechnology, part of the National Nanotechnology Coordinated Infrastructure Program, which is supported by the NSF grant NNCI-1542153. This work benefited from the use of the SasView application, originally developed under NSF award DMR-0520547. SasView contains code developed with funding from the European Union's Horizon 2020 research and innovation program under the SINE2020 project, grant agreement no. 654000. The authors also thank A. Pröschel and K. Mysore for their help in simulating structures of various sizes, and $\mathrm{Z}$. Wang and J. Fu for the SEM images of NP-Sb and NP-Cu.

\section{References}

1 J. Fu, E. Detsi and J. T. M. De Hosson, Surf. Coat. Technol., 2018, 347, 320-336.

2 Z. Deng and E. Detsi, Nanoscale, 2017, 9, 11858-11863.

3 E. Detsi, P. Onck and J. T. M. De Hosson, ACS Nano, 2013, 7, 4299-4306.

4 E. Detsi, M. S. Sellès, P. R. Onck and J. T. M. De Hosson, Scr. Mater., 2013, 69, 195-198.

5 K. Ma, J. S. Corsi, J. Fu and E. Detsi, ACS Appl. Nano Mater., 2018, 1, 541-546.

6 J. B. Cook, T. C. Lin, E. Detsi, J. N. Weker and S. H. Tolbert, Nano Lett., 2017, 17, 870-877.

7 E. Detsi, S. H. Tolbert, S. Punzhin and J. T. M. De Hosson, J. Mater. Sci., 2015, 51, 615-634.

8 S. Punzhin, E. Detsi, A. Kuzmin and J. T. M. De Hosson, J. Mater. Sci., 2014, 49, 5598-5605.

9 J. Fu, Z. Deng, T. Lee, J. S. Corsi, Z. Wang, D. Zhang and E. Detsi, ACS Appl. Energy Mater., 2018, 1, 3198-3205.

10 E. Detsi, S. Punzhin, J. Rao, P. R. Onck and J. T. M. De Hosson, ACS Nano, 2012, 6, 3734-3744.

11 E. Detsi, X. Petrissans, Y. Yan, J. B. Cook, Z. Deng, Y. L. Liang, B. Dunn and S. H. Tolbert, Phys. Rev. Mater., 2018, 2, 1-10.
12 J. B. Cook, H. S. Kim, T. C. Lin, S. Robbennolt, E. Detsi, B. S. Dunn and S. H. Tolbert, ACS Appl. Mater. Interfaces, 2017, 9, 19063-19073.

13 E. Detsi, J. B. Cook, B. K. Lesel, C. L. Turner, Y. L. Liang, S. Robbennolt and S. H. Tolbert, Energy Environ. Sci., 2016, 9, 540-549.

14 E. Detsi, Z. Vuković, S. Punzhin, P. M. Bronsveld, P. R. Onck and J. T. M. D. Hosson, CrystEngComm, 2012, 14, 5402-5406.

15 H. Yaghoobnejad Asl, J. Fu, H. Kumar, S. S. Welborn, V. B. Shenoy and E. Detsi, Chem. Mater., 2018, 30, 1815-1824.

16 E. Detsi, M. Salverda, P. R. Onck and J. T. M. De Hosson, J. Appl. Phys., 2014, 115, 044308.

17 A. Pröschel, J. Chacko, R. Whitaker, M. A. U. Chen and E. Detsi, J. Electrochem. Soc., 2019, 166, H146-H150.

18 E. Detsi, J. B. Cook, B. K. Lesel, C. L. Turner, Y. L. Liang, S. Robbennolt and S. H. Tolbert, Energy Environ. Sci., 2016, 9, 540-549.

19 M. Li, Z. Wang, J. Fu, K. Ma and E. Detsi, Scr. Mater., 2019, 164, 52-56.

20 K. Ma, Z. Wang, J. Fu, J. S. Corsi, S. S. Welborn, J. T. M. De Hosson and E. Detsi.

21 H. Yaghoobnejad Asl, J. Fu, H. Kumar, S. S. Welborn, V. B. Shenoy and E. Detsi, Chem. Mater., 2018, 30, 1815-1824.

22 J. B. Cook, E. Detsi, Y. Liu, Y. L. Liang, H. S. Kim, X. Petrissans, B. Dunn and S. H. Tolbert, ACS Appl. Mater. Interfaces, 2017, 9, 293-303.

23 E. Detsi, S. Punzhin, P. R. Onck and J. T. M. De Hosson, J. Mater. Chem., 2012, 22, 4588-4591.

24 J. B. Cook, E. Detsi, Y. Liu, Y. L. Liang, H. S. Kim, X. Petrissans, B. Dunn and S. H. Tolbert, ACS Appl. Mater. Interfaces, 2017, 9, 293-303.

25 J. Dendooven, K. Devloo-Casier, M. Ide, K. Grandfield, M. Kurttepeli, K. F. Ludwig, S. Bals, P. Van Der Voort and C. Detavernier, Nanoscale, 2014, 6, 14991-14998.

26 R. R. Salunkhe, Y. V. Kaneti and Y. Yamauchi, ACS Nano, 2017, 11, 5293-5308.

27 T. Li, S. Karwal, B. Aoun, H. Zhao, Y. Ren, C. P. Canlas, J. W. Elam and R. E. Winans, Chem. Mater., 2016, 28, 7082-7087.

28 A. C. Alba-Rubio, B. J. O'Neill, F. Shi, C. Akatay, C. Canlas, T. Li, R. Winans, J. W. Elam, E. A. Stach, P. M. Voyles and J. A. Dumesic, ACS Catal., 2014, 4, 1554-1557.

29 Z. Liu, N. Fujita, K. Miyasaka, L. Han, S. M. Stevens, M. Suga, S. Asahina, B. Slater, C. Xiao, Y. Sakamoto, M. W. Anderson, R. Ryoo and O. Terasaki, J. Electron Microsc., 2013, 62, 109-146.

30 R. E. Morris and P. S. Wheatley, Angew. Chem., Int. Ed., 2008, 47, 4966-4981.

31 E. Stavitski, M. Goesten, J. Juan-Alcañiz, A. MartinezJoaristi, P. Serra-Crespo, A. V. Petukhov, J. Gascon and F. Kapteijn, Angew. Chem., Int. Ed., 2011, 50, 9624-9628.

32 C. S. Tsao, M. S. Yu, T. Y. Chung, H. C. Wu, C. Y. Wang, K. Sen Chang and H. L. Chen, J. Am. Chem. Soc., 2007, 129, 15997-16004.

33 S. J. Yang, T. Kim, J. H. Im, Y. S. Kim, K. Lee, H. Jung and C. R. Park, Chem. Mater., 2012, 24, 464-470. 
34 C. Chen, J. Kim and W.-S. Ahn, Korean J. Chem. Eng., 2014, 31, 1919-1934.

35 S. T. Meek, J. A. Greathouse and M. D. Allendorf, Adv. Mater., 2011, 23, 249-267.

36 T. M. Davis, T. O. Drews, H. Ramanan, C. He, J. Dong, H. Schnablegger, M. A. Katsoulakis, E. Kokkoli, A. V. Mccormick, R. L. Penn and M. Tsapatsis, Nat. Mater., 2006, 5, 400-408.

37 L. R. A. Follens, A. Aerts, M. Haouas, T. P. Caremans, B. Loppinet, B. Goderis, J. Vermant, F. Taulelle, J. A. Martens and C. E. A. Kirschhock, Phys. Chem. Chem. Phys., 2008, 10, 5574-5583.

38 A. Aerts, L. R. A. Follens, M. Haouas, T. P. Caremans, M. A. Delsuc, B. Loppinet, J. Vermant, B. Goderis, F. Taulelle, J. A. Martens and C. E. A. Kirschhock, Chem. Mater., 2007, 19, 3448-3454.

39 P.-P. E. A. de Moor, T. P. M. Beelen, R. A. van Santen, K. Tsuji and M. E. Davis, Chem. Mater., 1999, 11, 36-43.

40 B. J. Smith, L. R. Parent, A. C. Overholts, P. A. Beaucage, R. P. Bisbey, A. D. Chavez, N. Hwang, C. Park, A. M. Evans, N. C. Gianneschi and W. R. Dichtel, ACS Cent. Sci., 2017, 3, 58-65.

41 D. Beaudoin, T. Maris and J. D. Wuest, Nat. Chem., 2013, 5, 830-834.

42 T. Ma, E. A. Kapustin, S. X. Yin, L. Liang, Z. Zhou, J. Niu, L. H. Li, Y. Wang, J. Su, J. Li, X. Wang, W. D. Wang, W. Wang, J. Sun and O. M. Yaghi, Science, 2018, 361, 48-52.

43 V. Presser, J. McDonough, S. H. Yeon and Y. Gogotsi, Energy Environ. Sci., 2011, 4, 3059-3066.

44 M. Oschatz, E. Kockrick, M. Rose, L. Borchardt, N. Klein, I. Senkovska, T. Freudenberg, Y. Korenblit, G. Yushin and S. Kaskel, Carbon N. Y., 2010, 48, 3987-3992.

45 Y. Gogotsi, A. Nikitin, H. Ye, W. Zhou, J. E. Fischer, B. Yi, H. C. Foley and M. W. Barsoum, Nat. Mater., 2003, 2, 591-594.

46 Y. Korenblit, M. Rose, E. Kockrick, L. Borchardt, A. Kvit, S. Kaskel and G. Yushin, ACS Nano, 2010, 4, 1337-1344.

47 J. Chmiola, P. L. T. Celine Largeot, P. Simon and Y. Gogotsi, Science, 2010, 328, 480-483.

48 W. Lu, D. Yuan, D. Zhao, C. I. Schilling, O. Plietzsch, T. Muller, S. Bräse, J. Guenther, J. Blümel, R. Krishna, Z. Li and H. C. Zhou, Chem. Mater., 2010, 22, 5964-5972.

49 P. Kuhn, A. Forget, D. Su, A. Thomas and M. Antonietti, J. Am. Chem. Soc., 2008, 130, 13333-13337.

50 Q. Zhang, E. Uchaker, S. L. Candelaria and G. Cao, Chem. Soc. Rev., 2013, 42, 3127-3171.

51 S. A. Ansari and Q. Husain, Biotechnol. Adv., 2012, 30, 512-523. 52 E. M. Bringa, J. D. Monk, A. Caro, A. Misra, L. Zepeda-Ruiz, M. Duchaineau, F. Abraham, M. Nastasi, S. T. Picraux, Y. Q. Wang and D. Farkas, Nano Lett., 2012, 12, 3351-3355.

53 X. Lang, L. Qian, P. Guan, J. Zi and M. Chen, Appl. Phys. Lett., 2011, 98, 093701.

54 F. Yu, S. Ahl, A. M. Caminade, J. P. Majoral, W. Knoll and J. Erlebacher, Anal. Chem., 2006, 78, 7346-7350.

55 S. Linic, P. Christopher and D. B. Ingram, Nat. Mater., 2011, 10, 911-921.
56 H. A. Chen, J. L. Long, Y. H. Lin, C. J. Weng and H. N. Lin, J. Appl. Phys., 2011, 110, 54302.

57 J. Biener, G. W. Nyce, A. M. Hodge, M. M. Biener, A. V. Hamza and S. A. Maier, Adv. Mater., 2008, 20, 1211-1217.

58 A. Wittstock, J. Biener and M. Bäumer, Phys. Chem. Chem. Phys., 2010, 12, 12919-12930.

59 V. Zielasek, B. Jürgens, C. Schulz, J. Biener, M. M. Biener, A. V. Hamza and M. Bäumer, Angew. Chem., Int. Ed., 2006, 45, 8241-8244.

60 H.-J. Qiu, H.-T. Xu, L. Liu and Y. Wang, Nanoscale, 2015, 7, 386-400.

61 N. Cheng, L. Ren, X. Xu, Y. Du and S. X. Dou, Adv. Energy Mater., 2018, 8, 1801257.

62 T. Fujita, P. Guan, K. McKenna, X. Lang, A. Hirata, L. Zhang, T. Tokunaga, S. Arai, Y. Yamamoto, N. Tanaka, Y. Ishikawa, N. Asao, Y. Yamamoto, J. Erlebacher and M. Chen, Nat. Mater., 2012, 11, 775.

63 Y. Ding, M. Chen and J. Erlebacher, J. Am. Chem. Soc., 2004, 126, 6876-6877.

64 C. Xu, Y. Liu, J. Wang, H. Geng and H. Qiu, J. Power Sources, 2012, 199, 124-131.

65 C. Xu, X. Xu, J. Su and Y. Ding, J. Catal., 2007, 252, 243-248.

66 T. Fujita, T. Tokunaga, L. Zhang, D. Li, L. Chen, S. Arai, Y. Yamamoto, A. Hirata, N. Tanaka, Y. Ding and M. Chen, Nano Lett., 2014, 14, 1172-1177.

67 Z. Liu and P. C. Searson, J. Phys. Chem. B, 2006, 110, 4318-4322.

68 E. Detsi, Z. G. Chen, W. P. Vellinga, P. R. Onck and J. T. M. De Hosson, J. Nanosci. Nanotechnol., 2012, 12, 4951-4955.

69 H. J. Jin and J. Weissmüller, Adv. Eng. Mater., 2010, 12, 714-723.

70 J. Biener, A. Wittstock, L. A. Zepeda-Ruiz, M. M. Biener, V. Zielasek, D. Kramer, R. N. Viswanath, J. Weissmüller, M. Bäumer and A. V. Hamza, Nat. Mater., 2009, 8, 47-51.

71 E. Detsi, P. R. Onck and J. T. M. De Hosson, Appl. Phys. Lett., 2013, 103, 193101.

72 O. Ruiz, M. Cochrane, M. Li, Y. Yan, K. Ma, J. Fu, Z. Wang, S. H. Tolbert, V. B. Shenoy and E. Detsi, Adv. Energy Mater., 2018, 8, 1801781.

73 S. L. Candelaria, Y. Shao, W. Zhou, X. Li, J. Xiao, J. G. Zhang, Y. Wang, J. Liu, J. Li and G. Cao, Nano Energy, 2012, 1, 195-220.

74 A. González, E. Goikolea, J. A. Barrena and R. Mysyk, Renewable Sustainable Energy Rev., 2016, 58, 1189-1206.

75 S. Liu, J. Feng, X. Bian, Y. Qian, J. Liu and H. Xu, Nano Energy, 2015, 13, 651-657.

76 E. Detsi, M. Van De Schootbrugge, S. Punzhin, P. R. Onck and J. T. M. De Hosson, Scr. Mater., 2011, 64, 319-322.

77 I. McCue, J. Stuckner, M. Murayama and M. J. Demkowicz, Sci. Rep., 2018, 8, 6761.

78 E. Detsi, E. De Jong, A. Zinchenko, Z. Vuković, I. Vuković, S. Punzhin, K. Loos, G. Ten Brinke, H. A. De Raedt, P. R. Onck and J. T. M. De Hosson, Acta Mater., 2011, 59, 7488-7497.

79 J. W. Kim, T. Wada, S. G. Kim and H. Kato, Scr. Mater., 2016, 122, 68-71. 
80 M. K. Khristosov, L. Bloch, M. Burghammer, Y. Kauffmann, A. Katsman and B. Pokroy, Nat. Commun., 2015, 6, 8841.

81 T. Kou, D. Li, C. Zhang, Z. Zhang and H. Yang, J. Mol. Catal. A: Chem., 2014, 382, 55-63.

82 Y. H. Tan, J. a. Davis, K. Fujikawa, N. V. Ganesh, A. V. Demchenko and K. J. Stine, J. Mater. Chem., 2012, 22, 6733-6745.

83 H. J. Qiu, L. Peng, X. Li, H. T. Xu and Y. Wang, Corros. Sci., 2015, 92, 16-31.

84 M. Mokhtari, C. Le Bourlot, J. Adrien, A. Bonnin, T. Wada, J. Duchet-Rumeau, H. Kato and E. Maire, Mater. Charact., 2018, 144, 166-172.

85 J. Erlebacher and I. McCue, Acta Mater., 2012, 60, 6164-6174.

86 R. D. MacPherson and D. J. Srolovitz, Nature, 2007, 446, 1053-1055.

87 J. Erlebacher, M. J. Aziz, A. Karma, N. Dimitrov and K. Sieradzki, Nature, 2001, 410, 450-453.

88 B. Chu and B. S. Hsiao, Chem. Rev., 2001, 101, 1727-1761.

89 I. S. Dmitri and H. J. K. Michel, Rep. Prog. Phys., 2003, 66, 1735.

90 A. Guinier and G. Fournet, Small angle scattering of $x$-rays, John Wiley \& Sons, Inc., New York, 1st edn, 1955.

91 A. J. Guinier, Proc. Phys. Soc., 1945, 57, 310-324.

92 W. Liu, W. L. Johnson, S. Schneider, U. Geyer and P. Thiyagarajan, Phys. Rev. B: Condens. Matter Mater. Phys., 1999, 59, 11755-11759.

93 D. P. Rolf, Nature, 1983, 302, 46-48.

94 A. Guinier, Nature, 1938, 142, 569-570.

95 A. Guinier, Ann. Phys., 1939, 11, 161-237.

96 O. Glatter and O. Kratky, Small Angle X-ray Scattering, London, 1982.

97 B. R. Pauw, J. Phys.: Condens. Matter, 2013, 25, 383201.

$98 \mathrm{~J}$. Als-Nielsen and D. McMorrow, Elements of modern X-ray physics, John Wiley \& Sons Ltd., West Sussex, 2nd edn, 2011.

99 P. Debye and A. M. Bueche, J. Appl. Phys., 1949, 20, 518-525.

100 P. Debye, H. R. Anderson and H. Brumberger, J. Appl. Phys., 1957, 28, 679-683.

101 O. Spalla, Chapter 3, Neutrons, X-rays and Light: Scattering Methods Applied to Soft Condensed Matter, North-Holland, 1st edn, 2002.

102 C. J. Dotzler, B. Ingham, B. N. Illy, K. Wallwork, M. P. Ryan and M. F. Toney, Adv. Funct. Mater., 2011, 21, 3938-3946.

103 S. H. Chen, S. L. Chang and R. Strey, J. Appl. Crystallogr., 1991, 24, 721-731.

104 M. Teubner, Europhys. Lett., 1991, 14, 403-408.

105 S. H. Chen, S. L. Chang, R. Strey, J. Samseth and K. Mortensen, J. Phys. Chem., 1991, 95, 7427-7432.

106 M. Peltomäki, G. Gompper and D. M. Kroll, J. Chem. Phys., 2012, 136, 134708.

107 K. V. Schubert, R. Strey, S. R. Kline and E. W. Kaler, J. Chem. Phys., 1994, 101, 5343-5355.

108 T. Sottmann, R. Strey and S.-H. Chen, J. Chem. Phys., 2002, 106, 6483-6491.

109 L. E. Scriven, Nature, 1976, 263, 123-125.
110 S. H. Chen, S. L. Chang and R. Strey, in Trends in Colloid and Interface Science IV, ed. M. Zulauf, P. Lindner and P. Terech, Steinkopff, Darmstadt, 2007, pp. 30-35.

111 N. F. Berk, Phys. Rev. A: At., Mol., Opt. Phys., 1991, 44, 5069-5079.

112 N. F. Berk, Phys. Rev. Lett., 1987, 58, 2718-2721.

113 M. Teubner and R. Strey, J. Chem. Phys., 1987, 87, 3195-3200.

114 S. Ichilmann, K. Rücker, M. Haase, D. Enke, M. Steinhart and L. Xue, Nanoscale, 2015, 7, 9185-9193.

115 E. S. Hatakeyama, B. R. Wiesenauer, C. J. Gabriel, R. D. Noble and D. L. Gin, Chem. Mater., 2010, 22, 4525-4527.

116 Y. Ito, H. J. Qiu, T. Fujita, Y. Tanabe, K. Tanigaki and M. Chen, Adv. Mater., 2014, 26, 4145-4150.

117 B. Krause, H. J. P. Sijbesma, P. Münüklü, N. F. A. Van Der Vegt and M. Wessling, Macromolecules, 2001, 34, 8792-8801.

118 S. Kim, M. Yoo, N. Kang, B. Moon, B. J. Kim, S. H. Choi, J. U. Kim and J. Bang, ACS Appl. Mater. Interfaces, 2013, 5, 5659-5666.

119 J. Erlebacher, R. C. Newman and K. Sieradzki, Nanoporous Gold: From an Ancient Technology to a High-Tech Material, The Royal Society of Chemistry, 2012, pp. 11-29.

120 J. W. Cahn, J. Chem. Phys., 1965, 42, 93-99.

121 M. Graf, B. N. D. Ngô, J. Weissmüller and J. Markmann, Phys. Rev. Mater., 2017, 1, 76003.

122 C. Soyarslan, S. Bargmann, M. Pradas and J. Weissmüller, Acta Mater., 2018, 149, 326-340.

123 I. McCue, E. Benn, B. Gaskey and J. Erlebacher, Annu. Rev. Mater. Res., 2016, 46, 263-286.

124 Y. Ding, Y. J. Kim and J. Erlebacher, Adv. Mater., 2004, 16, 1897-1900.

125 I. McCue, A. Karma and J. Erlebacher, MRS Bull., 2018, 43, 27-34.

126 J. Erlebacher, J. Electrochem. Soc., 2004, 151, C614.

127 J. Erlebacher and K. Sieradzki, Scr. Mater., 2003, 49, 991-996.

128 T. Fujita, L. H. Qian, K. Inoke, J. Erlebacher and M. W. Chen, Appl. Phys. Lett., 2008, 92, 251902.

129 Y. C. K. Chen-Wiegart, S. Wang, Y. S. Chu, W. Liu, I. McNulty, P. W. Voorhees and D. C. Dunand, Acta Mater., 2012, 60, 4972-4981.

130 Y. C. K. Chen-Wiegart, T. Wada, N. Butakov, X. Xiao, F. De Carlo, H. Kato, J. Wang, D. C. Dunand and E. Maire, J. Mater. Res., 2013, 28, 2444-2452.

131 K. Kolluri and M. J. Demkowicz, Acta Mater., 2011, 59, 7645-7653.

132 J. Erlebacher, Phys. Rev. Lett., 2011, 106, 225504.

133 I. McCue, B. Gaskey, P. A. Geslin, A. Karma and J. Erlebacher, Acta Mater., 2016, 115, 10-23.

134 P. A. Geslin, I. McCue, B. Gaskey, J. Erlebacher and A. Karma, Nat. Commun., 2015, 6, 1-19.

135 I. McCue, J. Snyder, X. Li, Q. Chen, K. Sieradzki and J. Erlebacher, Phys. Rev. Lett., 2012, 108, 225503.

136 H. Jinnai, Y. Nishikawa, S. Chen, S. Koizumi and T. Hashimoto, Phys. Rev. E, 2000, 61, 6673-6780.

137 S. H. Chen, D. D. Lee, K. Kimishima, H. Jinnai and T. Hashimoto, Phys. Rev. E: Stat. Phys., Plasmas, Fluids, Relat. Interdiscip. Top., 1996, 54, 6526-6531. 
138 H. Jinnai, T. Koga, Y. Nishikawa, T. Hashimoto and S. T. Hyde, Phys. Rev. Lett., 1997, 78, 2248-2251.

139 J. W. Cahn and J. E. Hilliard, J. Chem. Phys., 1958, 28, 258-267.

140 J. W. Cahn, Acta Metall., 1962, 10, 179-183.

141 J. W. Cahn, Acta Metall., 1961, 9, 795-801.

142 J. W. Cahn, Acta Metall., 1966, 14, 1685-1692.

143 I. Wolfram, Research, Mathematica, Wolfram Research, Inc., Version 11., 2018.

144 M. Kardar, Statistical Physics of Fields, Cambridge University Press, 2012.

145 S. M. Choi, S. H. Chen, T. Sottmann and R. Strey, Phys. A, 2002, 304, 85-92.

146 S.-H. Chen and S.-M. Choi, J. Appl. Crystallogr., 1997, 30, 755-760.

147 L. A. Feigin and D. I. Svergun, Structure Analysis by SmallAngle X-Ray and Neutron Scattering, 1987.

148 R. E. Winans and P. Thiyagarajan, Energy Fuels, 1988, 2, 356-358.

149 F. Gao, C. C. Ho and C. C. Co, Macromolecules, 2006, 39, 9467-9472.

150 B. Hammouda, J. Appl. Crystallogr., 2010, 43, 716-719.

151 G. Beaucage, J. Appl. Crystallogr., 1995, 28, 717-728.

152 D. Eliezer, P. A. Jennings, P. E. Wright, S. Doniach, K. O. Hodgson and H. Tsuruta, Science, 1995, 270, 487.

153 T. J. Prosa, B. J. Bauer, E. J. Amis, D. A. Tomalia and R. Scherrenberg, J. Polym. Sci., Part B: Polym. Phys., 1997, 35, 2913-2924.

154 K. Sinkó, V. Torma and A. Kovács, J. Non-Cryst. Solids, 2008, 354, 5466-5474.

155 E. Smorgonskaya, R. Kyutt, A. Danishevskii, C. Jardin, R. Meaudre, O. Marty, S. Gordeev and A. Grechinskaya, J. Non-Cryst. Solids, 2002, 299-302, 810-814.

156 É. A. Smorgonskaya, R. N. Kyutt, V. B. Shuman, A. M. Danishevskii, S. K. Gordeev and A. V. Grechinskaya, Phys. Solid State, 2002, 44, 2001-2008.

157 R. N. Kyutt, É. A. Smorgonskaya, A. M. Danishevskii, S. K. Gordeev and A. V. Grechinskaya, Phys. Solid State, 1999, 41, 1359-1363.

158 E. A. Chavez Panduro, T. Beuvier, M. Fernández Martínez, L. Hassani, B. Calvignac, F. Boury and A. Gibaud, J. Appl. Crystallogr., 2012, 45, 881-889.

159 G. Beaucage, J. Appl. Crystallogr., 1996, 29, 134-146.

160 R. Diduszko, A. Swiatkowski and B. J. Trznadel, Carbon N. Y., 2000, 38, 1153-1162.
161 K. Kaneko, M. Sato, T. Suzuki, Y. Fujiwara, K. Nishikawa and M. Jaroniec, J. Chem. Soc., Faraday Trans., 1991, 87, 179-184.

162 M. H. Reich, S. P. Russo, I. K. Snook and H. K. Wagenfeld, J. Colloid Interface Sci., 1990, 135, 353-362.

163 J. Teixeira, J. Appl. Crystallogr., 1988, 21, 781-785.

164 J. E. Martin and A. J. Hurd, J. Appl. Crystallogr., 1987, 20, 61-78.

165 Y. C. K. Chen, Y. S. Chu, J. Yi, I. McNulty, Q. Shen, P. W. Voorhees and D. C. Dunand, Appl. Phys. Lett., 2010, 96, 043122.

166 A. A. El-Zoka, J. Y. Howe, R. C. Newman and D. D. Perovic, Acta Mater., 2019, 162, 67-77.

167 M. Hakamada and M. Mabuchi, J. Mater. Res., 2009, 24, 301-304.

168 R. N. Viswanath, V. A. Chirayath, R. Rajaraman, G. Amarendra and C. S. Sundar, Appl. Phys. Lett., 2013, 102, 253101.

169 S. Kuwano-Nakatani, K. Uchisawa, D. Umetsu, Y. Kase, Y. Kowata, K. Chiba, T. Fujita, M. Chen, T. Tokunaga, S. Arai, Y. Yamamoto and N. Tanaka, Mater. Trans., 2015, 56, 468-472.

170 M. Hakamada and M. Mabuchi, Mater. Lett., 2008, 62, 483-486.

171 J. Wang, R. Xia, J. Zhu, Y. Ding, X. Zhang and Y. Chen, J. Mater. Sci., 2012, 47, 5013-5018.

172 M. Ziehmer, K. Hu, K. Wang and E. T. Lilleodden, Acta Mater., 2016, 120, 24-31.

173 K. Hu, M. Ziehmer, K. Wang and E. T. Lilleodden, Philos. Mag., 2016, 96, 3322-3335.

174 E. T. Lilleodden and P. W. Voorhees, MRS Bull., 2018, 43, 20-26.

175 C. A. Dreiss, K. S. Jack and A. P. Parker, J. Appl. Crystallogr., 2006, 39, 32-38.

176 D. S. Sivia, Elementary Scattering Theory for X-ray and Neutron Users, Oxford University Press, 2011.

177 S. R. Stock and B. D. Cullity, Elements of X-ray diffraction, 3rd edn, 2001.

178 C. T. Chantler, K. Olsen, R. A. Dragoset, J. Chang, A. R. Kishore, S. A. Kotochigova and D. S. Zucker, Detailed Tabulation of Atomic Form Factors, Photoelectric Absorption and Scattering Cross Section, and Mass Attenuation Coefficients for $Z=1-92$ from $E=1-10 \mathrm{eV}$ to $E=0.4-1.0 \mathrm{MeV}$, NIST, NIST Stand., 2005.

179 P. Brown, A. Fox, E. Maslen, M. A. O'Keefe and B. T. M. Willis, Int. Tables Crystallogr., 2004, C, 554-595.

180 SasView, https://www.sasview.org/. 